\title{
Central extracorporeal membrane oxygenation to lung transplantation after remote arterial switch operation
}

Adam Stephens, MD, ${ }^{a}$ Dean McKenzie, MD,${ }^{a}$ Jeffrey Heinle, MD, ${ }^{a}$ Ernestina Melicoff-Portillo, MD, Carolina Gazzaneo, MD, ${ }^{\mathrm{b}, \mathrm{c}}$ Ryan Coleman, MD, ${ }^{\mathrm{b}, \mathrm{c}}$ and Iki Adachi, MD, ${ }^{\mathrm{a}}$ Houston, Tex

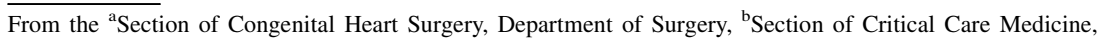
Department of Pediatrics, and ${ }^{\mathrm{c} S e c t i o n}$ of Pulmonary Medicine, Department of Pediatrics, Baylor College of Medicine, Houston, Tex.

Disclosures: The authors reported no conflicts of interest.

The Journal policy requires editors and reviewers to disclose conflicts of interest and to decline handling or reviewing manuscripts for which they may have a conflict of interest. The editors and reviewers of this article have no conflicts of interest.

Received for publication March 31, 2021; accepted for publication July 30, 2021; available ahead of print Aug 6, 2021.

Address for reprints: Adam Stephens, MD, Section of Congenital Heart Surgery, Department of Surgery, Baylor College of Medicine, 6651 Main Street, Legacy Tower, 19th Floor, MC 19345H, Houston, TX 77030 (E-mail: nastephe@texaschildrens.org).

JTCVS Techniques 2021;10:452-5

2666-2507

Copyright (c) 2021 The Author(s). Published by Elsevier Inc. on behalf of The American Association for Thoracic Surgery. This is an open access article under the CC BY-NC-ND license (http://creativecommons.org/licenses/bync-nd/4.0/).

https://doi.org/10.1016/j.xjtc.2021.07.020

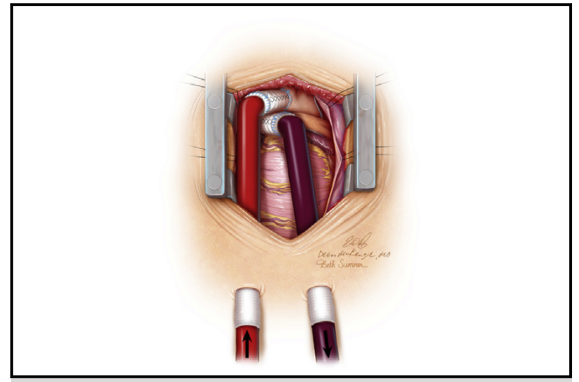

Central cannulation setup after Lecompte maneuver from remote arterial switch operation.

CENTRAL MESSAGE

Central extracorporeal membrane oxygenation with EXCOR (Berlin Heart Inc, The Woodlands, Tex) after remote arterial switch operation as a bridge to lung transplantation.

See Commentaries on pages 456 and 458. tients with end-stage lung disease to lung transplantation and allows for participation in physical therapy before transplantation. $^{4}$ Various peripheral cannulation strategies have been described for that purpose. Cannulation strategies may require special consideration if the scope of ECMO is not only for pulmonary support but also for resuscitation of a failing right ventricle (RV) in the setting of altered vascular anatomy after ASO. We describe a novel approach using the EXCOR cannulae (Berlin Heart Inc, The Woodlands, Tex) for central ECMO institution in a patient with idiopathic pulmonary arterial hypertension and severe RV failure after ASO.

\section{CASE REPORT}

This was a 13-year-old boy with of D-TGA who underwent an uncomplicated ASO with the traditional Lecompte maneuver performed (placing the pulmonary arteries (PA) anterior to the newly constructed posterior aorta) as a neonate at an outside institution. He presented to an outside hospital as a teenager with heart failure symptoms. Chest radiograph demonstrated massive cardiomegaly (Figure E1). Echocardiography revealed a dilated RV with severely depressed function, severe tricuspid regurgitation with a peak velocity of $\sim 7 \mathrm{~m} / \mathrm{s}$. The ventricular septum was bowing leftward during systole, causing near left ventricular cavity obliteration. In addition, there were small apical muscular ventricular septal defects with right-toleft shunting. Cross-sectional imaging did not identify a residual anatomic lesion related to his surgical repair that could account for his pulmonary hypertension.

Medical therapy was initiated with inotropic agents, oxygen, diuretics, and inhaled nitric oxide. Given the highrisk nature of his condition, the patient was transferred to our institution. Cardiac catheterization revealed suprasystemic PA and RV pressures with pulmonary vascular resistance of 34.2 Wood Units $/ \mathrm{m}^{2}$ and a transpulmonary gradient of $80 \mathrm{~mm} \mathrm{Hg}$. Alternative strategy such as Potts shunt was not considered due to his severe RV dysfunction. Subsequent medical review board approval for lung transplantation was granted. Despite maximum medical management, he declined clinically, developing presyncopal episodes, necessitating mechanical circulatory support. The plan was made for central ECMO as a bridge to lung transplantation using EXCOR cannulae for increased stability and durability to allow for optimal 
early mobility and ambulation in anticipation of potential extended time on the transplant waitlist.

A reoperative median sternotomy was performed. The RV was massively enlarged. Only the upper $1 \mathrm{~cm}$ of the ascending aorta was accessible due to the anteriorly relocated main PA (Figure 1). To overcome this obstacle, graft extensions were used to facilitate cannulation and allowed for anatomical cannulae alignment. An incision in the right upper quadrant of the abdomen was created to tunnel a $60^{\circ}$ 9-mm EXCOR arterial cannula to serve as an ECMO outlet. A second incision was created closer to the midline on the abdominal wall for an 85 9-mm EXCOR arterial cannula. After heparin administration, the right pulmonary artery (RPA) was controlled with a partial occlusion clamp to anastomose a 14-mm Gelweave graft (Terumo Aortic, Sunrise, Fla). The other end of the graft was then anastomosed to the $85^{\circ}$ cannula. A $14-\mathrm{mm}$ graft was beveled and anastomosed to the $60^{\circ}$ arterial cannula. The ascending aorta/ innominate artery junction was controlled with a partial occlusion clamp to anastomose another 14-mm Gelweave graft that had already been connected to the $60^{\circ}$ cannula. A longitudinal arteriotomy was created and the graft was sewn to the aortic incision (Figure 2).

After appropriate connection of the cannulae to the circuit, ECMO support was initiated. There were no anatomic

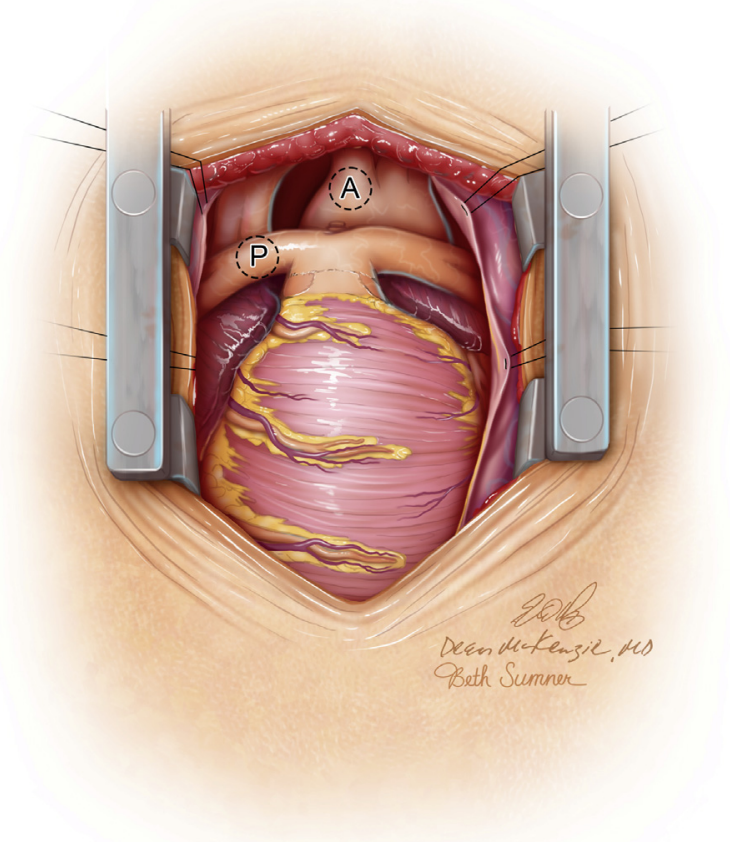

FIGURE 1. Cardiac anatomy after previous arterial switch operation with Lecompte with planned cannulation sites labeled. A, Aorto-innominate artery junction; $P$, right pulmonary artery.

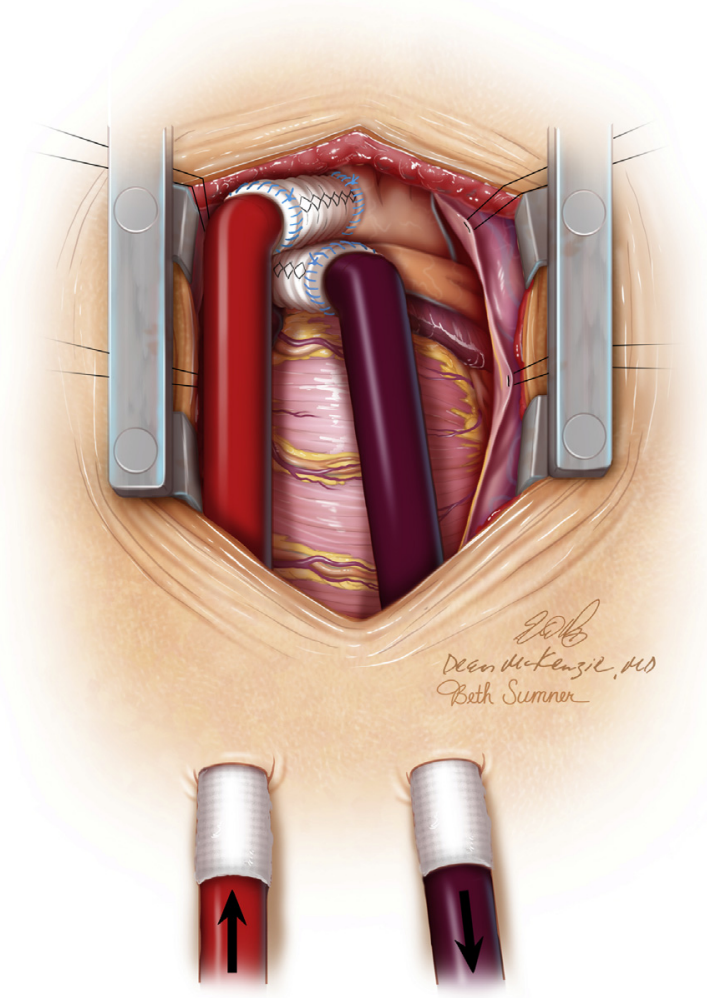

FIGURE 2. A unique cannulation strategy for the distal ascending aortainnominate artery outflow graft and the right pulmonary artery inflow graft after the Lecompte maneuver from previous arterial switch operation.

changes with the graft extensions upon ECMO initiation, with inlet pressures remaining positive due to $\mathrm{PAH}$, indicating no real potential for graft collapse. Hemodynamics remained stable with sternal closure. Postoperative chest radiograph demonstrated good cannula positioning with sternal closure (Figure E2). On postoperative day 1, he was taken back to the operating room for bleeding from the aortic/innominate artery anastomotic site. No complications occurred thereafter. He was extubated on postoperative day 2.

Over the next 2 weeks, he transitioned to room air and participated in daily rehabilitation efforts that included walking treadmill exercises, physical therapy, and enteral nutrition. ECMO settings between postoperative day 3 to transplant were stable requiring minimal manipulation throughout his post-ECMO pretransplant rehabilitation period. Consistently, flow was between 65 and $81 \mathrm{cc} / \mathrm{kg} /$ $\min (81 \mathrm{cc} / \mathrm{kg} / \mathrm{min}$ achieved $100 \%$ flow rate for this patient per institutional guidelines), sweep $0.3 \mathrm{~L} / \mathrm{min}$, inspired oxygen fraction $80 \%$, bivalirudin 0.55 to $0.59 \mathrm{mg} / \mathrm{kg} / \mathrm{h}$, which is commonly used at this institution for anticoagulation with durable mechanical circulatory support. Partial thromboplastin time hepzyme levels of 55 to 65 are targeted. There was no echocardiographic evidence of any unusual flow 
pattern or distribution anomalies related to this cannulation strategy.

Eighteen days later, suitable organs were identified and accepted. Due to the innominate artery cannulation and extreme cardiomegaly, a reoperative sternotomy was performed to gain optimal upper mediastinal exposure. There were significant mediastinal adhesions, likely due to previous bleeding at the innominate artery-chimney graft interface but the pleural adhesions were reasonable. The existing innominate graft was extended with a new 14$\mathrm{mm}$ graft to be used for cardiopulmonary bypass perfusion. The cannula on the RPA was used for venous drainage. Having ascertained adequate anticoagulation, full-flow bypass initiated with this configuration (PA drainage and aorta-innominate arterial return). This allowed for cardiac decompression, facilitating safer dissection and subsequent transition to bicaval cannulation. His particular anatomy provided several challenges. The hilum was not accessible due to massive cardiomegaly. For this reason, pneumonectomy on the left side as well as implantation was performed under cardiac arrest. On the right side, pneumonectomy was performed with the heart beating but implantation required a second period of cardiac arrest for adequate exposure. Cardiopulmonary bypass was weaned off with excellent graft function. Total bypass time was 9 hours and 51 minutes with an aortic crossclamp time of 4 hours and 13 minutes and donor ischemic time of 6 hours and 27 minutes. Both aortic-innominate and right PA chimney grafts were cut short and suture closed before chest closure. Blood product administration included platelet $429 \mathrm{~mL}$ and packed red blood cells $350 \mathrm{~mL}$. He was extubated on postoperative day 2 and discharged to inpatient rehabilitation 25 days later in good condition. Predischarge echocardiography demonstrated mildly depressed RV function and mild tricuspid regurgitation with a peak velocity of $\sim 3 \mathrm{~m} / \mathrm{s}$.

The authors obtained informed written consent from the patient's guardian for the publication of the study data.

\section{DISCUSSION}

ASO has become the surgical standard of care for treatment of D-TGA, with greatly improved outcomes in the last 3 decades as experience and surgical techniques continue to evolve and improve. ${ }^{5}$ Patients with idiopathic PAH after this operation have the potential to clinically decompensate despite aggressive medical therapies leading to lung transplantation as a final option. ${ }^{3}$ It is well described that pretransplant respiratory rehabilitation and participation in physical therapy, ideally with ambulation, significantly improves transplant candidacy. ${ }^{4}$

For this patient, a novel approach was used to support his RV failure and rapid clinical decompensation. Central ECMO with EXCOR cannulae in the RPA (inlet) to innominate artery (outlet) configuration provided RV afterload reduction allowing for functional RV recovery. The cuffed component of the cannulae at the skin exit sites with the chest closed made it more suitable for aggressive physical rehabilitation than traditional forms of peripheral ECMO support via the neck. It allowed for early mobilization, ambulation, and participation in a more rigorous physical therapy program, improving transplant candidacy. The decompensating patient with PAH post-ASO can have a severely enlarged right heart, making sternal re-entry and dissection challenging. The Lecompte maneuver alters the normal hilar anatomy and obscures much of the ascending aorta leaving a short, sometimes large anterior main PA. To overcome these challenges, the arterial inflow and outflow cannulation strategies were adjusted accordingly, as previously described. The configuration of RPA as the inflow to aorta as the outflow serves to decompress the pulmonary vascular bed, reduce $\mathrm{RV}$ afterload and provides a better substrate for functional RV recovery.

In this case, massive cardiomegaly with altered vascular anatomy after ASO in a failing RV provided multiple obstacles. Using Gelweave graft extensions allowed for cannulation in a challenging anatomical environment. This form of central ECMO support provided adequate support for a failing RV and allowed for sternal closure and early physical activity and successful bridge to lung transplant. In addition to functional RV recovery, the previously seen cardiomegaly was dramatically reduced in size, as shown in the 7-month follow-up chest radiograph (Figure E3).

This demonstrates that using EXCOR cannulae with the configuration that has been described here provides a stable, durable form of central mechanical support in the failing RV due to PAH. It allows for early extubation and prompt initiation of both respiratory and physical rehabilitation, improving both transplant candidacy and the potential for a successful postoperative outcome. In addition, it serves to leap over the anatomic hurdles that come with the patient with refractory idiopathic PAH post-ASO in need of urgent mechanical circulatory support as a bridge to lung transplantation.

\section{References}

1. Jatene AD, Fontes VF, Paulista PP, Souza LC, Neger F, Galantier M, et al. Anatomic correction of transposition of the great arteries. J Thorac Cardiovasc Surg. 1976;72:364-70.

2. Zijlstra WM, Elmasry O, Pepplinkhuizen S, Ivy DD, Bonnet D, Luijendijk P, et al. Pulmonary arterial hypertension in children after neonatal arterial switch operation. Heart. 2017;103:1244-9.

3. Watanabe T, Adachi O, Suzuki Y, Notsuda H, Niikawa H, Matsuda Y, et al. Lung transplant for pulmonary arterial hypertension in children after neonatal arterial switch operation. Ann Thorac Surg. 2015;100:e133-4.

4. Tipograf Y, Salna M, Minko E, Grogan EL, Agerstrand C, Sonett J, et al. Outcomes of extracoporeal membrane oxygenation as a bridge to lung transplantation. Ann Thorac Surg. 2019;107:1456-63.

5. Jensen HA, Ntsinjana HN, Bull C, Brown K, Taylor AM, Kostolny M. Performance monitoring of the arterial switch operation: a moving target. EurJ Cardiothorac Surg. 2015;48:716-23. 


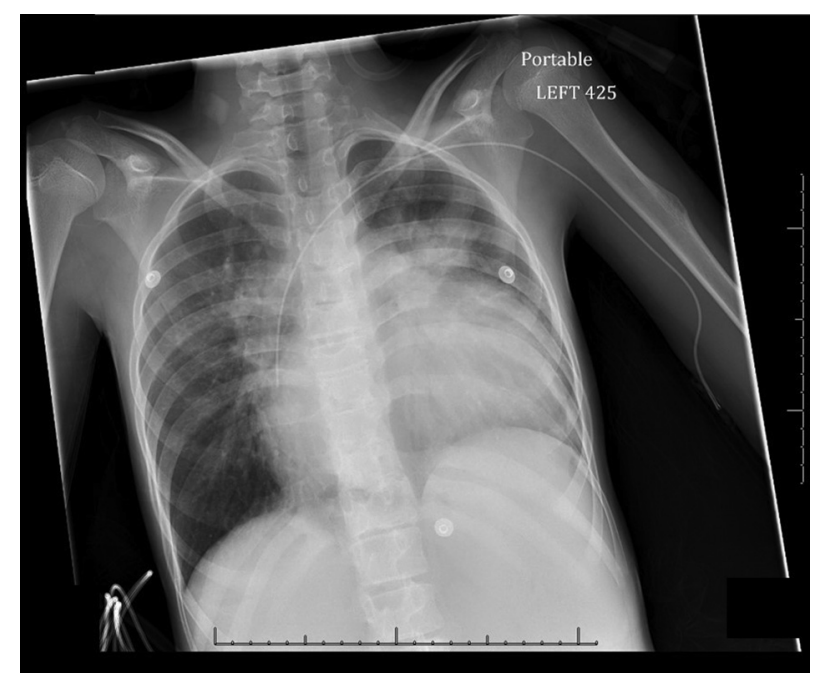

FIGURE E1. Preoperative chest radiograph demonstrating massive cardiomegaly.

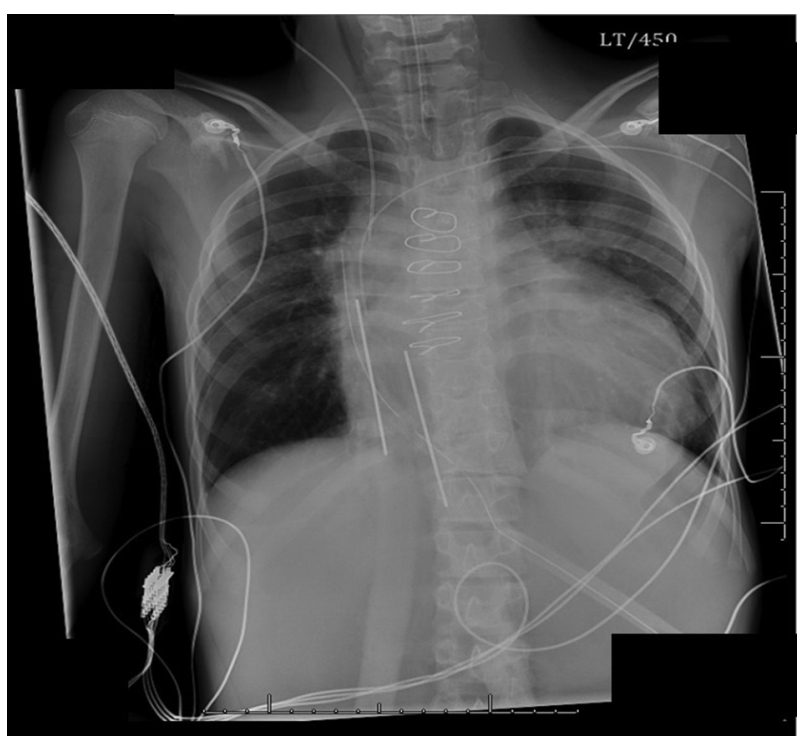

FIGURE E2. Immediate postoperative chest radiograph displaying cannula placement.

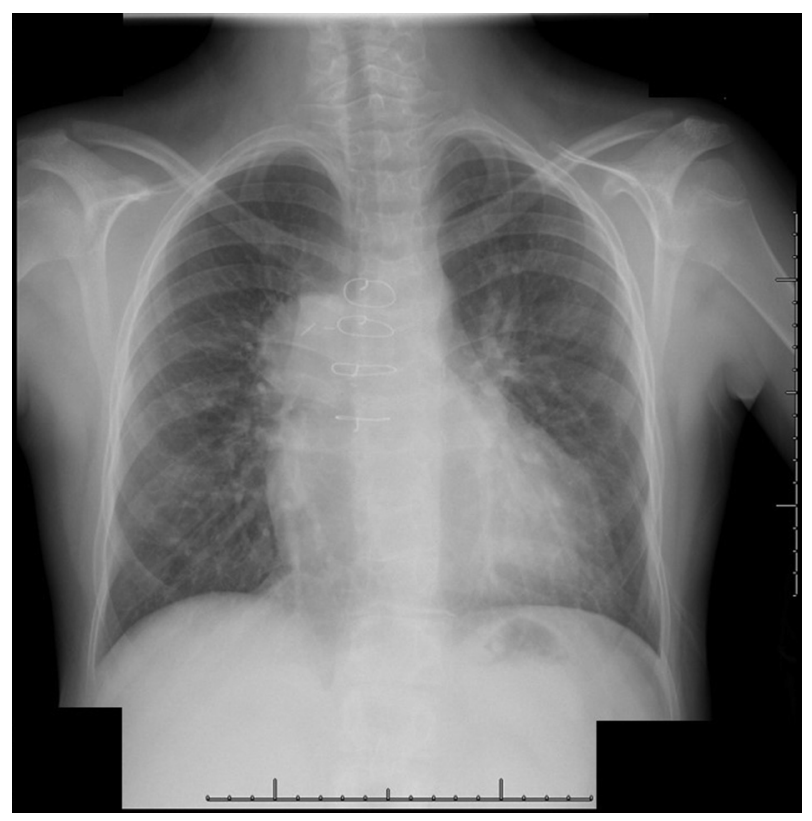

FIGURE E3. Seven-month follow-up chest radiograph displaying marked reduction in cardiac size. 